\title{
Common operation metrics for storage ring light sources
}

\author{
A. Lüdeke* \\ PSI, 5232 Villigen, Switzerland \\ M. Bieler \\ DESY, Notkestraße 85, 22607 Hamburg, Germany \\ R. H. A. Farias \\ LNLS, Caixa Postal 6192, CEP 13083-970, Campinas - São Paulo, Brazil \\ S. Krecic \\ Elettra-Syncrotrone Trieste, SS14, Km 163.5, 34149 Basovizza TS, Italy \\ R. Müller \\ HZB, BESSY II, Albert-Einstein-Str. 15, 12489 Berlin, Germany \\ M. Pont \\ CELLS-ALBA Synchrotron, Carrer de la Llum 2-26, 08290 Cerdanyola del Vallès, Barcelona, Spain \\ M. Takao \\ JASRI/SPring-8, 1-1-1, Kouto, Sayo-cho, Sayo-gun, Hyogo 679-5198, Japan \\ (Received 13 March 2015; revised manuscript received 12 July 2016; published 4 August 2016)

\begin{abstract}
Storage ring light sources aim for high operational reliability. Very often beam availability is used as an operation metric to measure the reliability. A survey of several light sources reveals that the calculation of availability varies significantly between facilities. This complicates useful comparisons of reliability. Furthermore the beam availability does not provide insight regarding reliability of beam characteristics such as orbit and beam size stability. The authors propose specific metrics to evaluate the reliability of storage ring light sources; these metrics allow a detailed and meaningful comparison across facilities. Such comparisons are useful to further optimize the reliability of storage ring light source facilities.
\end{abstract}

DOI: 10.1103/PhysRevAccelBeams.19.082802

\section{INTRODUCTION}

Reliability is typically defined as the ability of a system to serve a given function over time. In this sense the reliability of a particle accelerator is very important for user facilities like storage ring light sources and provides an important design objective for new large scale accelerator facilities as for example the International Linear Collider [1]. Clearly operation metrics should quantify the reliability of a particle accelerator. If the objective is to assess the improvement of a specific facility over time, then the operation metric should be closely related to specific user requirements [2]. In order to compare reliability of different facilities, one needs a common standard for the calculation of operation metrics.

\footnotetext{
*andreas.luedeke@psi.ch

Published by the American Physical Society under the terms of the Creative Commons Attribution 3.0 License. Further distribution of this work must maintain attribution to the author(s) and the published article's title, journal citation, and DOI.
}

Commonly beam availability or "up-time" is often used to compare the reliability of light sources. While statistics are published for most light sources, few facilities supply precise definitions on how the information is calculated. A survey of several light sources revealed [3] that the calculation of this metric varies considerably. The conditions under which beam is considered "available" are often defined only in simple common sense terms, and even if there are formal definitions, they differ between facilities. Furthermore a large variety of failure modes is often convolved into "beam not available," commonly referred to as "downtime." Since many downtime failure modes are specific to individual facilities, this further complicates the comparison of beam availability.

Our aim is to provide simple, well-defined, formal operation metrics for storage ring light sources to make beam reliability at these facilities comparable. The metrics serve to clarify for each facility the beam parameters specified for the users, how the statistical data is processed and how well the standards are met. The authors make the case that the application of these metrics will refine the 
ability to learn from and compare reliability at storage ring of light sources.

\section{CURRENT STATUS}

The definition of "beam availability" is an important metric useful to compare operation at different facilities. A survey on failure analysis in 2008 at nine different light sources [4] revealed significant differences for the calculation of beam availability [3]. In many cases the beam availability calculations were determined by identifying events as downtime that interrupted data acquisition for the majority of the synchrotron radiation beam users. Some facilities considered "long" injector outages—causing "decaying beam" operation-to be downtime, others accounted for these events individually. Most facilities only counted "beam available" between two successive outages if it exceeded a minimum duration. The minimal required duration varied between 15 and 60 minutes. In cases of long beam outages, most facilities organized compensation time for users to finish experiments. The compensation time was also accounted for in different ways, depending on the facility: some fully subtracted the extra beam time from the downtime, whilst others ignored this extra time for the beam availability calculation. All light sources recorded events other than beam outages, such as increased beam size or orbit problems, but no facility published statistics on these failure modes at regular intervals.

During a discussion round at the ARW 2013 in Melbourne [5] we polled the calculation of beam availability from participants representing ten light sources [6] and obtained a similar result as the survey of 2008 .

Based on this data the authors concluded that a direct comparison of accelerator reliability is currently difficult if not impossible. It is in the interest of every facility, from operations to management, to be able to assess accelerator reliability as compared with other facilities. Internally this is important to support plans for upgrades and maintenance. It can also assist the decision making process when information relative to other facilities is available. Comparisons of reliability may also serve as a trigger for strong interlaboratory collaborations. If a particular failure mode is identified to be more frequent at multiple facilities, this provides incentive for common efforts to develop more reliable solutions. As a consequence of using common metrics, clear comparisons may also emerge when requesting funding from supranational authorities.

\section{THE TASK}

After comparing the current situation at different facilities, we discussed the advantages and disadvantages of alternate definitions for downtime and beam availability. The comparison soon revealed that local metrics for beam availability are often used to assess the development of the facility over time. Every change to that metric could potentially compromise the ability of comparison with historic data. In addition, local definitions of beam availability are often part of contracts and changes to the definition would require a change of contract. We therefore propose common operation metrics in addition to existing definitions; metrics that do seek to not redefine existing terms such as beam availability and downtime.

Currently many failure modes are categorized under the general term downtime. We propose to disentangle those failure modes and record statistical data for each mode individually. Our aim is to define a list of failure modes; to have data published concerning number and duration of these failure modes at different light sources-including data for the operation schedule of each facility-to be able to normalize the failure data across storage ring light source facilities.

The definition for each failure mode should be adaptable to the different character of light sources because nominal beam parameters differ and variations of specific parameters may have consequences at one facility and not others. The definitions aim to clearly show what kind of stability can be expected at each facility. The statistical data for the different failure modes will then reveal how reliable the parameters have been in the past. In addition, the limits for each failure mode are shown to depend on the specific operation mode of the storage ring light source. If a facility is operated at different energies or with different filling specifications, then every operation mode can have facilityspecific limits.

\section{FACILITY OPERATION MODES}

Storage ring based light sources can be operated in a variety of different modes. Some modes are optimized for high photon flux (e.g. multibunch fillings) while other modes create short pulses (e.g. single-bunch and low- $\alpha$ operation). Some sources only operate in a single mode and others switch from mode to mode for specific users on a weekly basis. The total amount of annual beam time is of course facility dependent.

Many failure modes defined at a given facility depend on the specific operational mode. The total beam current in single-bunch operation for instance would be considered insufficient for useful multibunch operation at the same facility.

In the following we will illustrate the differences presenting data for seven facilities: ALBA, BESSY II, Elettra, LNLS-UVX, PETRA III, SPring-8 and SLS. A reference publication is provided for each light source.

Table I shows an overview of the scheduled operation user time and user modes.

\section{A. ALBA}

The ALBA light source [7] presently runs with one operation mode: $100 \mathrm{~mA}$ multibunch. 
TABLE I. Overview of operation in 2014 (PETRA III numbers for 2013, since 2014 was shut down).

\begin{tabular}{lccl}
\hline \hline Facility & User time $(\mathrm{h})$ & Modes & \multicolumn{1}{c}{ Comments } \\
\hline ALBA & 3888 & 1 & Multibunch, top-up \\
BESSY II & 5403 & 4 & Multibunch, single bunch, low- $\alpha$ or "PTB" \\
Elettra & 5083 & 2 & Multibunch hybrid, beam energies 2.0 and $2.4 \mathrm{GeV}$ \\
LNLS-UVX & 3816 & 2 & Multibunch and single bunch \\
PETRA III & 5812 & 4 & 40 to 960 bunches at $100 \mathrm{~mA}$ \\
SPring-8 & 3360 & 9 & Variety of different fill modes \\
SLS & 4984 & 1 & Multibunch hybrid, top-up \\
\hline \hline
\end{tabular}

The fill pattern consists of ten bunch trains each with 32 bunches and a 24 ns gap in between. Since September 2014 ALBA operates in top-up mode, before that it was decay mode. In top-up mode the beam decays down to $98.5 \mathrm{~mA}$ and is then reinjected to $100 \mathrm{~mA}$. In the decay mode ALBA injected $120 \mathrm{~mA}$ twice a day and let the beam decay to $72 \mathrm{~mA}$ in between.

ALBA scheduled $3888 \mathrm{~h}$ for users in 2014. Extra user time has been provided for compensation in some rare cases of long machine outages.

\section{B. BESSY II}

A standard week of scheduled user beam time at BESSY II [8] starts Tuesday 7:00 a.m. and ends Sunday 23:00 providing the three basic user operation modes: multibunch hybrid, single-bunch or low- $\alpha$. The user modes are as follows.

(i) Multibunch hybrid mode comprises $299 \mathrm{~mA}$ total current, kept constant by top-up injections. General bunch pattern is an even filling of 300 bunches and a gap of 100 bunches (200 ns). In addition to this multibunch current, five specific bunches serve dedicated experiments (see below). The size of the gap around each camshaft bunch is variable to support pulse picking with a mechanical chopper.

The five specific bunches are: one camshaft bunch at higher current $(4 \mathrm{~mA})$ inserted in the middle of the (purity controlled) gap for pump-probe experiments; three slicing bunches of $4 \mathrm{~mA}$ each can be located opposite to the gap, sequentially sliced with $6 \mathrm{kHz}$ repetition, 20 fs laser pulses, generating 100 fs x-ray pulses; and one pseudosingle bunch typically three buckets away from the end of the gap, which is resonantly excited for pseudosingle-bunch experiments.

In this standard mode two backup modes for degraded beam conditions are possible. BESSY can easily switch to microtron injection in case of LINAC problems. Then multibunch top-up is feasible, occasional refilling of the custom bunches requires a decay phase and a closure of the beam line shutters. If the top-up lifetime constraint $>5 \mathrm{~h}$ cannot be met (e.g. due to vacuum problems) BESSY can set a lower nominal current of $\approx 250 \mathrm{~mA}$ to stay operational.

(ii) The single-bunch mode consists of $14 \mathrm{~mA}$ in a (purity controlled) single bunch, refilled with top-up, used for time resolved experiments ( $2-3$ weeks per year). This mode depends on the LINAC, since the microtron can inject single bunches only with low efficiency, thus a lasting LINAC failure results in a decaying beam degraded mode.

(iii) In low- $\alpha$ mode an even filling of either $100 \mathrm{~mA}$ (short pulse 5 mode) or $15 \mathrm{~mA}$ ( $\mathrm{THz}$ mode, nonbursting coherent synchrotron radiation) is provided in an alternating, 12 hour period decaying beam sequence (2-3 weeks per year).

(iv) National Bureau of Standards (PTB) mode provides specific beam conditions according to their experimental requirements.

BESSY II provided 5403 hours of user operation in 2014. Extra user time is provided only in specific cases of hardship, like coincidence of a week long failure of essential components and rare operating conditions in demand. More frequently the weekly machine development shifts are provided for user operation as so-called "bonus time."

\section{Elettra}

Elettra [9] operates for about $75 \%$ of user dedicated time at $2 \mathrm{GeV}$ while for the remaining $25 \%$ at $2.4 \mathrm{GeV}$; being the only facility to operate at two energies in top-up. The main operating modes are multibunch with a dark gap of $90 \mathrm{~ns}$ and hybrid (at $20 \%$ of the total user beam time) with a single bunch in the middle of the dark gap. The operating intensities are $310 \mathrm{~mA}$ at $2 \mathrm{GeV}$ and $160 \mathrm{~mA}$ at $2.4 \mathrm{GeV}$ with a $5 \mathrm{~mA}$ single bunch added when in hybrid mode. Multibunch and single bunch are refilled by top-up with a delta of $1 \mathrm{~mA}$.

Elettra scheduled 5083 hours of user operation in 2014. Users are compensated for downtime when possible. This extra user time is usually announced three days in advance.

\section{LNLS-UVX}

The UVX at LNLS [10] operates in two modes: $100 \%$ filling (148 buckets), and single bunch. LNLS-UVX operates with low energy injection and the beam is delivered in the decay mode. In a typical run the initial current is $250 \mathrm{~mA}$, injected at $500 \mathrm{MeV}$ and ramped up to $1.37 \mathrm{GeV}$, the nominal operation energy. Injection takes 
place twice a day and is scheduled to last 30 minutes at most. By the end of the run, after about 11 hours, the current decays down to $130 \mathrm{~mA}$. Single-bunch shifts are available since 2003, but the provision of these shifts depends on the demand by the users. In the single-bunch mode the initial current is typically in the order of $9 \mathrm{~mA}$ and is less interesting for high-flux, multibunch users. Operation in hybrid mode is impractical due to the characteristics of the UVX injection system.

In 2014 LNLS-UVX was operated 3816 hours for users. Since UVX is operated from Monday to Saturday, it is common practice to provide bonus user time on the weekend in case of operational problems during the week; a total of 87 hours bonus time was provided in 2014 .

\section{E. PETRA III}

PETRA III [11], with a circumference of $2.3 \mathrm{~km}$ and an rf of $500 \mathrm{MHz}$, has a harmonic number of 3840. For about $50 \%$ of the user time PETRA III is operated in a continuous filling mode; the rest is used for timing modes. All modes are in top-up operation at $100 \mathrm{~mA}$. In the continuous filling modes either every fourth bucket (960 bunches), or every eighth (480 bunches) is filled; in timing mode either 40 bunches (192 ns bunch spacing) or 60 bunches (128 ns bunch spacing) are filled.

PETRA III operated 5812 hours in 2013 and went into a long shutdown in February 2014 for an expansion project. Users have not been compensated for long beam outages so far.

\section{F. SPring-8}

SPring-8 [12] runs in nine operation modes, one of which is multibunch with twelve 160 bunch trains, also several-bunch modes and hybrid modes. The severalbunch modes consist of equally spaced bunches (or bunch trains), i.e. 203 bunches, 84 trains of four bunches, and 29 trains of 11 bunches. The hybrid modes are composed of partially filled multibunch trains and isolated single bunches, i.e. 11/29-filling +1 bunch, 1/7-filling +5 bunches, $1 / 14$-filling +12 bunches, $2 / 29$-filling +26 bunches, and $4 / 58$-filling +53 bunches. The impurity of an isolated bunch is maintained under $10^{-8}$ in top-up operation.

The scheduled user time in 2014 was 3360 hours. Users are not compensated for long beam outages.

\section{G. SLS}

The Swiss Light Source (SLS) [13] runs only in $80 \%$ filling mode (390 out of 480 buckets), $400 \mathrm{~mA}$ top-up. A single bunch in the gap with a higher charge can be added on demand, the so-called "camshaft mode." Typically the single bunch is filled to 4 times the charge of the other buckets. Switching between the modes does not affect users of the $80 \%$ filling, since the beam current is kept constant at $400 \mathrm{~mA}$ in both.

A total of 4984 hours of user operation was scheduled in 2014. Only 4824 hours were prescheduled for user experiments. Two blocks of 80 hours were scheduled in addition to allow to compensate users for longer outages. Those backup user shifts were assigned to user experiments roughly a month in advance, and were given to those users who were not able to complete their experiments. Two beam availability numbers are published: with and without taking backup shift compensation into account. The compensated beam availability is mainly used for in-house purposes and aims to be always above $100 \%$. Infrequently part of the weekly machine development time is provided for user experiments, to compensate for recent machine problems. These shifts are not accounted for in the operation statistics.

\section{DEFINING PRIMARY FAILURE MODES}

Reliability is the ability of a system to provide a certain function continuously over time. The function of a light source is to provide synchrotron radiation for user experiments. From the perspective of a user it does not matter, what specific type of failure prevented performing an experiment. But in order to progress to a high reliability light source, one needs to differentiate failure modes; for each mode a solution should be found to increase the meantime-between-failure, and to minimize the mean-time-torecover.

The beam current in a storage ring is the primary parameter for beam delivery. Based on the beam current one can define two simple failure modes: no-beam and "low-beam-current" (see Fig. 1). These two modes are discussed in the next sections with examples. Beam limits

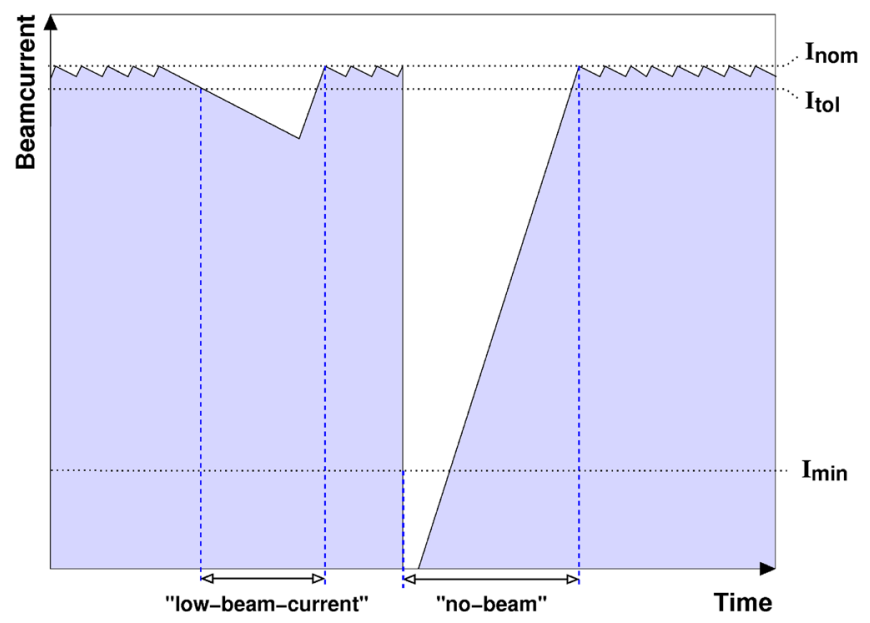

FIG. 1. A "no-beam" failure starts when the beam current drops below the current $I_{\min }$; a "low beam current" already starts below the current limit $I_{\text {tol }}$. Both modes stop when the nominal beam current $I_{\text {nom }}$ is recovered. 
$I_{\text {nom }} \geq I_{\text {tol }} \geq I_{\min }$ can readily be defined for each operation mode of a facility.

Further failure modes will be discussed in the next section.

\section{A. No-beam}

When the beam current is below $I_{\min }$ the no-beam failure mode starts. It stops when the nominal beam current $I_{\text {nom }}$ is reached again.

\section{B. Low-beam-current}

This failure mode starts when the beam current drops below $I_{\text {tol }}$, but only if the machine is not in the no-beam failure mode (otherwise all no-beam failures would be low-beam-current failures as well). The low-beam-current failure stops when the beam current reaches the nominal beam current $I_{\text {nom }}$ again. For facilities in top-up mode the current limit $I_{\text {tol }}$ should be slightly below the current to start top-up injections. For non-top-up facilities $I_{\text {tol }}$ is below the typical beam current when a refill would be scheduled.

\section{Primary failure modes in practice}

Table II shows the current limits for the primary failure modes, as they are used today, partly depending on the operation modes at the seven facilities. The column $I_{\text {nom }}$ shows the maximum current in the given operation mode. The current $I_{\text {inj }}$ is the typical value when injection would start in this operation mode, below $I_{\text {tol }}$ it would be considered a low-beam-current failure. The column $I_{\min }$ shows the condition under which a no-beam failure would start.

No-beam failures are defined to start when the beam current drops below a given limit. For several facilities this is currently not the case. ALBA, BESSY-II and LSLS-UVX consider a closure of the photon shutters by an interlock equivalent to a loss of the electron beam. For some facilities a no-beam failure stops only when the interlock is cleared that prevents the beam line shutters from opening (ALBA, BESSY II, LNLS-UVX, SPring-8) or when the insertion devices move back to their closed positions (SLS). PETRA III adds an amount of time to allow for the warm-up of the optical components at the beam lines. These practices are oriented to use a beam availability understood as photon beam availability. Here facilities count the time the photon beam is ready to be used at the beam lines.

Low-beam-current failures vary significantly between facilities. At Spring-8, for example, a beam decay of about $0.1 \%$ starts a low-beam-current failure, while at BESSY-II it starts only at $9 \%$ beam decay. The reason for this difference comes from the required current stability for the experiments. LNLS-UVX and PETRA III do not account for low-beam-current failures at all $\left(I_{\mathrm{tol}}=I_{\min }\right)$.

\section{Discussion of primary failure modes}

A downtime often only ends after the photon shutter or insertion device control is given back to the users, which is not compatible with the no-beam rule. This can be solved by having additional failure types for "photon-shutterclosed" or "insertion-devices-blocked-open" when there is not a no-beam failure. The application of this metric will disentangle failures of different nature. A former downtime at ALBA would then be the sum of no-beam and photonshutter-closed failures. It would allow a better comparison to facilities like the SLS that do not have a global photon shutter interlock.

If several failures are recorded for a single incident, then it should be recorded if one failure was preceded by another: if the photon shutters are interlocked, the beam is then dumped and afterwards it takes five minutes until the insertion devices are unblocked, then it should be visible from the failure data that these were not three but rather only one interruption of user operation.

The authors agreed to allow arbitrary limits for no-beam failures; in practice this does not make a large difference: situations are rare where the stored current drops from the nominal beam current to less than $50 \%$ but not to zero.

Low-beam-current failures vary significantly with the definition of the $I_{\text {tol }}$ limit. Nevertheless comparing the tolerated beam decay is a useful way to judge current stability at a facility. For those facilities where the limits are

TABLE II. Primary failure mode limits.

\begin{tabular}{lccccl}
\hline \hline Facility & Operation mode & $I_{\text {nom }}(\mathrm{mA})$ & $I_{\text {inj }}(\mathrm{mA})$ & $I_{\text {tol }}(\mathrm{mA})$ & \multicolumn{1}{c}{$I_{\text {min }}$} \\
\hline ALBA & Decay & 120.0 & 72.0 & 72.0 & Photon shutter $(\mathrm{PhS})$ \\
ALBA & Top-up & 100.0 & 98.5 & 95.0 & PhS \\
BESSY II & MB & 299.0 & 299.0 & 272.0 & PhS or $200 \mathrm{~mA}$ \\
BESSY II & SB & 14.0 & 13.5 & 12.0 & PhS or $8 \mathrm{~mA}$ \\
Elettra & $2.0 \mathrm{GeV}$ & 310.0 & 309.0 & 307.5 & $0 \mathrm{~mA}$ \\
Elettra & $2.4 \mathrm{GeV}$ & 160.0 & 159.0 & 158.0 & $0 \mathrm{~mA}$ \\
LNLS-UVX & Decay & 250.0 & 130.0 & 60.0 & PhS or $60 \mathrm{~mA}$ \\
PETRA III & Top-up & 101.0 & 100.0 & 75.0 & $75 \mathrm{~mA}$ \\
SPring-8 & Top-up & 99.5 & 99.5 & 99.4 & $0 \mathrm{~mA}$ \\
SLS & Top-up & 402.0 & 400.0 & 399.0 & $20 \mathrm{~mA}$ \\
\hline \hline
\end{tabular}


similar, the failure rates allow a meaningful comparison of the reliability of the injection process.

\section{SECONDARY FAILURE MODES}

The two primary beam-current failure modes are easy to measure, but are not always sufficient to determine if the beam is usable for experiments. In this section we define secondary failure modes to categorize other common problems of storage ring operation. In contrast to the primary failure modes, most of the secondary failure modes are often not easy to measure at all facilities or for all operation modes. Failure data for these modes are rarely published, and therefore common metrics that fit all facilities are difficult to define. Here we provide examples of possible secondary metrics for these failure modes.

Table III shows a comparison of secondary failure modes: if they are on-line recorded at the different facilities and which failure modes are reported in yearly statistics to the public.

\section{A. Distorted orbit}

A stable electron beam orbit is a prerequisite for most experiments. A possible failure mode definition might be to record orbit deviations above $20 \%$ of the transverse beam size. This would require a different limit for each beam position monitor. We rather suggest a simpler definition: to require the rms orbit distortion to stay below a nominal value $d x_{\text {nom }}, d y_{\text {nom }}$ for each facility and operation mode.

$A L B A$ for instance provides to the beam lines the rms orbit distortion in both the horizontal and the vertical plane. The beam lines are informed whenever this deviation is out of specification. Data at the photon beam position monitors is also used as a figure of merit for the orbit and beam lines are informed if the beam position at the source point deviates from nominal by more than $20 \%$. Orbit feedback outages are recorded by the operator and can be crosschecked with a log file generated by the slow orbit feedback which registers any interruption to the feedback. Operators generate a beam incidence entry in the logbook for each feedback interruption.

BESSY II covers all "orbit-out-of-specification" situations as a "distorted-orbit" failure: if none of the orbit feedbacks is usable (orbit feedback outage) or if the rms deviation from nominal exceeds $0.08 \mathrm{~mm}$. Typical rms orbit deviation ranges between $0.00-0.01 \mathrm{~mm}$. Orbitfeedback outages are recorded if they last longer than $60 \mathrm{sec}$. Succeeding failures are counted as one if the feedback runs for less than $10 \mathrm{~min}$.

Elettra has currently a long term orbit stability ( 2 to 5 days) of $\pm 5 \mu \mathrm{m}$ maximum deviations while the short term stability ( 24 hours) is $2 \%$ of the beam size $(1.7 \mu \mathrm{m}$ horizontally and $1.2 \mu \mathrm{m}$ vertically). Globally the absolute rms orbit distortion must stay below $400 \mu \mathrm{m}$ in the horizontal plane and $300 \mu \mathrm{m}$ in the vertical plane. Typical values are rms x $330 \mu \mathrm{m}$ and rms y $250 \mu \mathrm{m}$. Orbit feedback outages are recorded if they last longer than $10 \mathrm{sec}$.

$L N L S-U V X$ records orbit distortions that exceed $10 \%$ of the beam size in any plane, measured relative to a beambased-alignment defined "golden orbit." The beam lines are informed about the distortion and a fault event is recorded. The limits are vertical $\pm 8 \mu \mathrm{m}$ and horizontal $\pm 30 \mu \mathrm{m}$.

PETRA III has a fast orbit feedback. If this feedback fails and prespecified limits for the orbit deviation are exceeded, the beam is dumped automatically to protect the machine. At the insertion devices the limits for a beam dump are $\pm 250 \mu \mathrm{m}$ deviation from the nominal orbit in the vertical plane or $\pm 500 \mu \mathrm{m}$ in the horizontal plane. A warning is issued if the beam position or the angle of the beam deviates from the nominal orbit by more than a limit which is of the order of $\pm 5 \mu \mathrm{m}$ or $\pm 0.5 \mu \mathrm{rad}$ in the vertical plane and $\pm 15 \mu \mathrm{m}$ or $\pm 2 \mu \mathrm{rad}$ in the horizontal plane. Orbit related beam dumps are investigated to find the root cause of the deviation (i.e. faulty magnet power supply or a glitch in the orbit feedback).

SPring-8 stabilizes closed-orbit deviations to the submicron level by orbit feedback acting once per second. Abrupt changes of the orbit, for example by gap changes of an insertion device, are corrected by this feedback.

The most sensitive users desire variations of the photon beam pointing direction below 1 microradian. This corresponds to $\pm 10 \mu \mathrm{m}$ in the horizontal and $\pm 5 \mu \mathrm{m}$ in the vertical plane.

Monthly variation of the closed-orbit distortion grows to almost this value in both planes. These slow variations are ignored, and only abrupt orbit changes are noted in the logbook.

TABLE III. Secondary failure modes at different facilities.

\begin{tabular}{lccccc}
\hline \hline Facility & Distorted orbit & Low-beam lifetime & Beam blowup & Distorted fill & Short up-time (h) \\
\hline ALBA & On-line & On-line & On-line & $\ldots$ & 0.5 \\
BESSY II & On-line & On-line & On-line & On-line & 1.0 \\
Elettra & On-line & On-line & On-line & $\ldots$ & 1.0 \\
LNLS-UVX & On-line & On-line & On-line & $\ldots$ & 1.0 \\
PETRA III & On-line & $\ldots$ & $\ldots$ & $\ldots$ & $\leq 1$ \\
SPring-8 & On-line & On-line & On-line & On-line & 0 \\
SLS & Report & On-line & On-line & On-line & 1.0 \\
\hline \hline
\end{tabular}


TABLE IV. Distorted-orbit failure mode for different facilities, covering orbit feedback outages and deviations from the nominal orbit.

\begin{tabular}{lll}
\hline \hline Facility & \multicolumn{1}{c}{ Distortion } & \multicolumn{1}{c}{ Remark } \\
\hline ALBA & Max $+20 \%$ or feedback off & Recorded \\
BESSY II & $\operatorname{rms}>3 \mu \mathrm{m}$ or off & Recorded \\
Elettra & $\operatorname{rms}>1.7_{x},>1.2_{y} \mu \mathrm{m}$ or off & Recorded \\
LNLS-UVX & $\operatorname{Max}>30_{x},>8_{y} \mu \mathrm{m}$ & Recorded \\
PETRA III & $\operatorname{Max}>500_{x},>250_{y} \mu \mathrm{m}$ & $B$ dumped \\
PETRA III & $\operatorname{Max}>15_{x},>5_{y} \mu \mathrm{m}$ or off & Warning issued \\
SPring-8 & Max $>10_{x},>5_{y} \mu \mathrm{m}$ & Fast change recorded \\
SLS & Feedback off & Recorded and published \\
\hline \hline
\end{tabular}

SLS records and evaluates all outages of the fast orbit feedback which are longer than 10 seconds. If all beam position monitors are used within the orbit feedback, then the deviation is always "zero" as long as the fast orbit feedback is running with $250 \mu$ s cycle time. Large transient or persistent closed-orbit deviations will switch off the orbit feedback to avoid beam loss due to malfunctioning beam position monitors. Succeeding outages are counted as one if the feedback runs for less than 2 minutes. Number and duration of the orbit-feedback outages are reported in the yearly operation statistics.

Table IV shows a comparison of how distorted-orbit failures are handled at the different facilities.

Distorted-orbit failures are recorded at most facilities but are rarely taken into account in the yearly failure statistics. The limits when an orbit is considered out of specification are varying by orders of magnitude between different facilities. Publication of these limits and the associated failure rates would be very useful to compare facilities.

\section{B. Low-beam-lifetime}

Facilities operating in top-up mode keep the beam current constant even with a low-beam-lifetime. This can cause an increased frequency of injections and therefore more distortions and background radiation for the experiments. The limit for a low-beam-lifetime mode depends on the facility and the specific operation mode. We propose to define a minimal lifetime $\tau_{\text {low }}$ for each user operation mode at a facility and record whenever the lifetime is below that limit for more than a minute.

$A L B A$ has a nominal beam lifetime that is given by the combination of filling pattern, radio frequency (rf) voltage and transverse coupling constant. The typical lifetime at $100 \mathrm{~mA}$ is 22 hours. ALBA operates with six rf cavities each fed by two inductive output tubes (IOTs), and a typical "low-beam-lifetime" failure is caused by the trip of one IOT. Since it is the trip of a subsystem, the operator will record it. Normally the operator will recover the IOT and thus recover the nominal beam lifetime. A beam lifetime below 18 hours is considered "low." Top-up is stopped if the lifetime drops below 10 hours.

$S L S$ has a typical beam lifetime of about 8 hours. Depending on the vacuum conditions and the selected coupling this lifetime may vary in practice between 6 and 10 hours during normal operation. A low-beam-lifetime leads to more frequent injections for top-up. A low-beamlifetime failure is automatically recorded when the beam lifetime stays below 4.5 hours for longer than five minutes. The failure mode stops as soon as the lifetime is above 4.5 hours for longer than one minute.

The failure mode low-beam-lifetime is not independently recorded at BESSY II, Elettra, LNLS-UVX, PETRA III or SPring-8 facilities.

Table V shows a comparison of what would be considered a low-beam-lifetime at the different facilities.

TABLE V. Low-beam-lifetime limits.

\begin{tabular}{|c|c|c|c|c|}
\hline Facility & Mode & $\tau_{\text {normal }}(\mathrm{h})$ & $\tau_{\text {low }}(\mathrm{h})$ & Remark \\
\hline ALBA & Decay & 22 & 18 & \\
\hline ALBA & Top-up & 22 & 18 & \\
\hline BESSY II & $\mathrm{MB}$ & 7 & 5 & Stops top-up \\
\hline BESSY II & SB & 2 & 1 & Stops top-up \\
\hline Elettra & $2.0 \mathrm{GeV}$ & 23 & 10 & \\
\hline Elettra & $2.4 \mathrm{GeV}$ & 27 & 10 & \\
\hline LNLS-UVX & Decay & $>14$ & Not defined & \\
\hline PETRA III & Continuous & 12 & Not defined & \\
\hline PETRA III & Timing & 1.5 & Not defined & \\
\hline SPring-8 & All modes & $15-50$ & 10 & \\
\hline SLS & Top-up & 8 & 4.5 & Yearly evaluation \\
\hline
\end{tabular}


TABLE VI. Beam-blowup limits.

\begin{tabular}{lccl}
\hline \hline Facility & Typical size $(\mu \mathrm{m} \times \mu \mathrm{m})$ & Blowup & Remark \\
\hline ALBA & $70 \times 30$ & $+20 \%$ & Recorded \\
BESSY II & $250 \times 14$ & $+30 \%$ & Recorded \\
Elettra & $260 \times 10$ & $+10 \%$ & No on-line measurement yet \\
LNLS-UVX & $380 \times 80$ & $+10 \%$ & Recorded \\
PETRA III & $140 \times 7$ & $\ldots$ & No on-line measurement \\
SPring-8 & $100 \times 12$ & $\ldots$ & Not an independent failure mode \\
SLS & $50 \times 10$ & $+50 \%$ & Recorded and evaluated \\
\hline \hline
\end{tabular}

Low-beam-lifetime failures are apparently rare events at most facilities. The example of ALBA shows that it still makes sense to record and evaluate these faults. The normal beam lifetime varies considerably between facilities and operation modes. The nominal beam lifetime at the SLS would be considered a very low lifetime at ALBA or SPring-8. But a significant decrease in the beam lifetime can cause problems at most facilities and should therefore be recorded to evaluate the reliability of the facility in this respect.

\section{Beam blowup}

The horizontal beam size should stay constant at a light source, since the emittance is intrinsically constant. We propose to define vertical and horizontal beam size limits for each operation mode and record whenever the beam dimensions are larger than these limits for more than a minute.

While this failure mode is easy to define, it is very hard to detect for many facilities. A beam height of $10 \mu \mathrm{m}$ requires costly diagnostics to measure it continuously to $10 \%$ precision.

Table VI shows a comparison of the "beam-blowup" failure mode detection.

Beam-blowup failures are again infrequent at most facilities. Betatron coupling can affect the occurrence of this failure type, since at very low coupling even small errors can lead to relatively large changes of the vertical beam size. For some facilities the vertical beam size is difficult to measure. Nevertheless one needs to define limits for the tolerated variation of the beam size. The number of reported failures outside these limits would be an essential measure for the reliability of the facility.

\section{Distorted filling and bunch purity}

Some experiments have very strict requirements on the ratio between a filled single bunch and the residual charge in neighboring buckets. This again depends on the specific requirements for each experiment. Deviations from the desired bunch filling may cause problems. This failure mode is mainly relevant to time resolved measurements and the usefulness of any definition depends on the requirements of the specific users. For each operation mode an allowed maximum charge deviation $d Q_{\max }$ should be defined.

A bunch purity of $10^{-8}$ requires a lengthy procedure to be measured to high precision. It is therefore not feasible to measure bunch purity continuously. The filling pattern can be measured continuously to an accuracy of several percent, but the diagnostics are only available at some light sources.

Table VII shows a comparison of the distorted-filling limits.

"Distorted-filling and bunch-purity" faults are not relevant at all facilities. Time resolved measurements depend on bunch purity. At present only few facilities have the means to measure the bunch purity on-line. Sophisticated procedures are required to measure a bunch charge ratio of $10^{-8}$. Many facilities do have the means to detect deviations from the nominal bunch charge distribution. Where these means exist we encourage to publish failure limits and associated data.

\section{E. Beam unrelated}

Some failures do not affect the beam, but they do affect user experiments. For instance if the beam is stored and all beam parameters are within the desired limits, there still can be problems that prevent users from running any

TABLE VII. Distorted-filling limits for different facilities.

\begin{tabular}{lcl}
\hline \hline Facility & Bunch charge deviation & \multicolumn{1}{c}{ Remark } \\
\hline ALBA & $\ldots$ & Planned for 2015 \\
BESSY II & $10 \%$ & Recorded \\
Elettra & $\ldots$ & No on-line measurement \\
LNLS-UVX & $\ldots$ & No on-line measurement \\
PETRA III & $\ldots$ & No on-line measurement \\
SPring-8 & $10 \%$ & Recorded, deviation after accumulation neglected \\
SLS & $100 \%$ & Feedback outages recorded \\
\hline \hline
\end{tabular}


experiments. Infrastructure outages like massive control system and IT-infrastructure failures or photon shutter interlocks can lead to these situations. There cannot be a simple rule to calculate the start and stop for all failures of this type; but they should be recorded if they have an influence on a significant number of the experiments.

Currently beam unrelated incidences are considered to be downtime at some facilities, if they prevent all beam lines to continue their measurements. This is the case at ALBA, Elettra and the SLS. Other facilities neglect these types of failures for their downtime calculation, as long as the electron beam was not affected, for example PETRA III. At most facilities these failures are evaluated on a case by case basis: for example an interlock of all photon shutter would clearly be considered downtime, at least at ALBA, BESSY II and LNLS-UVX; but a problem with the IT infrastructure might not, even if the majority of the users where affected.

"Beam-unrelated" failures should be recorded whenever they have an impact on a significant number of beam lines. These faults are often facility specific.

\section{F. Short user time}

Many facilities have a cutoff for a minimal time to store the beam. For example if less than one hour is recorded between two beam trips the time in between is counted as downtime. This can be defined as an extra failure mode: "short user time." The limit of what time is too short for user experiments depends on the time the facility needs to get into thermal equilibrium and on the length of measurement time at an experiment. Each facility should define this cutoff time limit $T_{\text {short user time; }}$; it may depend on the operation mode.

BESSY II, Elettra, LNLS-UVX and the SLS consider a beam delivery of a total length of less that one hour to be downtime; at ALBA the cutoff is at 30 minutes. PETRA III does not record short user time as separate fault criteria, but covers this by the rule which adds up to one hour or the length of the downtime to each beam outage. SPring- 8 does not have a cutoff for a beam delivery time.

Most facilities subtract short user time in the beam availability calculations; none currently records it as a separate failure mode. An independent recording would enable to calculate beam availability with and without accounting for the short user time. This would improve the comparability between facilities that handle short user time differently.

\section{SCHEDULE STATISTICS}

Some facilities are very flexible to compensate users for long beam outages. Either some of the machine development shifts are sacrificed after a beam outage, or prescheduled blocks of shifts are used to reschedule users when problems with the accelerator prevent them from finishing their experiments. These compensation times are currently accounted for in very different ways, depending on the facility.

\section{A. Proposed user time statistics}

We propose to define a set of a unified metrics to account for user operation times. User operation should be distinguished in two categories: (i) scheduled user time: time allocated for user experiments at least one month in advance; (ii) user backup time: time that was originally not allocated for experiments, but was later provided to reschedule users that could not finish their measurements.

The sum of these is the user time; operation failures should be recorded during all of this time. If scheduled user time is converted into shutdown time less than one month before this user time, then this time has to be recorded as a no-beam failure. This rule takes care that all beam outages are visible in the statistics: some facilities do not count outages of several days for the beam availability, because they reschedule all users to a newly allocated time and declare the downtime to be a shutdown time. This is very good for the users: better to be rescheduled than losing all beam time. But it prevents the comparison with facilities that handle such outages differently.

The following example will illustrate the proposed scheduling procedure: A facility has 4000 hours scheduled user time. A vacuum leak occurs in the storage ring and causes a beam outage of seven days. Management decides to declare these seven days to be shutdown time and to shorten the following shutdown time in two weeks time by seven days to reschedule all users.

According to our proposal this will be accounted for as a seven day or 168 hours no-beam event. The extra seven days are user backup time. The total user time will be 4168 hours. This still allows each facility to calculate beam availability But at the same time it provides statistical data that can be compared to other facilities.

\section{B. Discussion of user time statistics}

A clear and dependable accounting of the user operation time is a prerequisite for a meaningful operation reliability evaluation of each accelerator. To draw an extreme picture, if all beam outages are declared to be shutdowns retroactively then the beam is always $100 \%$ available during user operation. It is important that only those times can be counted as user time where the failures are recorded and evaluated. If failure modes within the extra time for the users are not analyzed, then this time must not be accounted as user time in the common operation metrics data.

At BESSY II the extra time given to the users has traditionally been provided with an unmanned control room during the night. In this mode small failures can lead to long outages. These failures have been counted separately, since it was a different operation mode. This allows one to evaluate the accelerator reliability for the scheduled time 
and the extra user time separately. By user request BESSY changed this policy in 2015. Now all usable beam time is manned by operations staff.

\section{FAILURE MODE STATISTICS}

\section{A. Numbers to be published}

Every facility should publish the number and total duration for each applicable primary and secondary failure mode over a year. In addition the user time according to Sec. VII should be published. These figures would allow a direct comparison of the operational reliability across synchrotron light sources.

\section{B. Standardized derived statistics}

Each facility can of course continue to calculate beam availability statistics according to their own internal standards to evaluate the evolution of their facility over time. But at the same time the standardized failure mode data would allow one to calculate a variety of standardized beam availabilities. Typical examples include "common metrics for overall beam availability during user time" and a "common metric compensated beam availability":

$$
\begin{aligned}
\text { Availability }_{\mathrm{CM}-\text { overall }} & =\frac{\left(T_{\text {user }}-\sum \text { no-beam }\right)}{T_{\text {user }}} \\
\text { Availability }_{\mathrm{CM}-\text { compensated }} & =\frac{\left(T_{\text {user }}-\sum \text { no-beam }\right)}{T_{\text {scheduled user }}} .
\end{aligned}
$$

Equation (1) calculates the beam availability to compare the reliability of different accelerators from the perspective of an accelerator physicist: how reliably the accelerator operates. Equation (2) is important to compare the facilities from a user perspective: did all scheduled experiments receive sufficient beam time.

The statistics of the secondary failure modes can be calculated independently, or several failure modes can be convolved into combined macrostatistics. At the SLS all interruptions of the beam, of the top-up or of the orbit feedback are convolved into a mean-time-betweendistortions. It would be very interesting for the SLS to compare their performance with other facilities. With the currently published failure data, this is not possible. If other facilities would publish failure data according to the common operation metrics, then these numbers could be used to calculate a mean-time-between-distortions for all facilities and compare to the SLS. The important aspect here is that derived statistics could be calculated in identical ways for all facilities, thus allowing comprehensive comparison across facilities.

\section{DISCUSSION}

Operation managers need to prioritize maintenance and upgrades, and to make efficient use of the available resources. Trends in operation metrics are important criteria to assign priorities to these tasks. Multidimensional metrics like the common operation metrics proposed here would allow a more comprehensive evaluation of priorities. If an upgrade of the injector would help to reduce the number of low-beam-current failures then a detailed statistics of injector faults is important to justify the upgrade and a comparison to the low-beam-current failure rate at other facilities could help to justify the need for improvement.

Common operation metrics data from other light sources could further help to assess the validity of a specific measure: it could demonstrate how similar measures at other facilities helped to improve specific failure modes; and the current failure rates can be directly compared to other facilities.

Existing statistics like beam availability may be continued to be used, to illustrate how a facility evolves over time, or if they are part of contracts. In addition it would be possible to calculate values for a standardized definition of beam availability at all facilities that publish their common metrics data.

The proposed common metrics do require an effort to record a larger number of failure modes at each facility. An automated recording of those failures would be beneficial to achieve accurate and reproducible accounting.

The authors consider no-beam, low-beam-current and distorted-orbit to be the most important failure modes for storage ring light sources. The detection of "beam blowup" is also important, but many facilities still lack the equipment to continuously measure the vertical beam size down to levels that matter to users. Every facility should publish the number and duration of "short-uptime" failures; those failures are already detected but if they would be published it would facilitate comparison of operation statistics from different light sources.

Some failure modes are considered of secondary priority. The low-beam-lifetime is easy to measure but often not a significant failure mode for machines running in top-up. Lifetime limits would be very low and actual events rare. A "distorted-fill" failure is mostly relevant for timing modes; the bunch purity levels required at some facilities can often not be measured in parallel with user operation, which prohibits automatic recording. The failure mode "beam-unrelated" is too broadly defined for an automated recording. The authors suggest that if certain types of this failure mode-such as an interlock to the beam line photon shutters-is recurring at a facility, a specific failure mode for this type should be recorded.

The authors are aware that successful user beam time depends on many factors, such as availability and dependability of the data acquisition system. Nevertheless the availability of photon beams with specified parameters on sample is the common prerequisite. This paper attempts to 
characterize significant quality and quantity losses to the available beam time in unambiguous ways, so they can be accounted for and compared. This is done from the perspective of a small number of very different light sources. If a number of facilities publish data according to this proposal, it would permit meaningful comparisons of reliability, a significant step forward. This is a first attempt to standardized definitions for operational reliability for storage ring light sources. For comprehensive analysis specifics of operational modes and resulting failures and their severity have to be completed for every facility.

Storage ring light sources are simple to operate compared to linac driven free-electron lasers: the number of operation modes is much smaller, particle beams in equilibrium are easier to understand, and beam stability is not as much influenced by stochastic fluctuations. Therefore detailed analysis of failure modes for less complex storage ring facilities may help to understand how the failure modes at more complex facilities can be defined and what factors will influence the operation reliability of these facilities.

\section{CONCLUSION}

The performance of storage ring light sources has been increasing for the past decades. Diffraction limited light sources aim now to provide extremely brilliant and highly coherent x-ray beams for experiments. This has the consequence that the requirements for orbit- and beamcurrent stabilization as well as for fluctuations in the beam size are getting tighter. State-of-the-art operation metrics must take these conditions into account: the performance requirements have to be strictly monitored and all events where the facility fails to meet requirements should be recorded, analyzed and published.

We propose simple, distinct and standardized operation metrics for storage ring light sources. These metrics allow a useful and detailed comparison of the reliability of different light sources. The primary operation metrics are easy to measure and clearly defined. Proposed secondary metrics have less immediate goals to create equal definitions for all light sources, but are intended to encourage the publication of additional statistics for these failure modes. Further discussion will be required to evolve towards a standardized set of rules to calculate a set of secondary operation metrics; the web page [14] provides information on the ongoing discussion. We are convinced that the proposed standard operation metrics will allow meaningful comparisons of reliability between storage ring light sources in contrast to the current nonstandard set of statistics.

[1] T. Himel, J. Nelson, N. Phinney, and M. Ross, Availability and reliability issues for ILC, in Proceedings of the 22nd Particle Accelerator Conference, Albuquerque, New Mexico (IEEE, New York, 2007).

[2] A. Lüdeke, Finding your happy-user-index, in Proceedings of the International Particle Accelerator Conference (JACoW, Geneva, 2014), pp. 3816-3818, http://accelconf. web.cern.ch/AccelConf/IPAC2014/papers/thpri024.pdf.

[3] A. Lüdeke, Operation event logging system of the Swiss light source, Phys. Rev. ST Accel. Beams 12, 024701 (2009).

[4] Survey participants for the failure analysis questionnaire came from APS, ESRF, SPring-8, Diamond, SOLEIL, BESSY II, Elettra, ANKA and SLS.

[5] D. McGilvery, Web page of the accelerator reliability workshop (2013), http://accelerators.org.au/indico/ conferenceDisplay.py? confId $=55$.

[6] Participants of the ARW'13 presenting beam availability calculations were from ALBA, Australian Synchrotron, BESSY II, Diamond, SPEAR, NSRRC, SOLEIL, Elettra, SLS and PETRA III.

[7] G. García and S. Ferrer, The Spanish light source ALBA, Synchrotron Radiat. News 27, 30 (2014).

[8] R. Bakker, Status and commissioning-results of BESSY II, in Proceedings of the 18th Particle Accelerator Conference, New York, 1999 (IEEE, New York, 1999).

[9] A.C.E. Karantzoulis and S. Krecic, Elettra status and future prospects, in Proceedings of the International Particle Accelerator Conference (JACoW, Geneva, 2015), http://accelconf.web.cern.ch/AccelConf/IPAC2015/ papers/tupwa051.pdf.

[10] A. R. D. Rodrigues, A. F. Craievich, and C.E. T. G. da Silva, Commissioning and operation of the first Brazilian synchrotron light source, J. Synchrotron Radiat. 5, 1157 (1998).

[11] E. Weckert, K. Balewski, W. Brefeld, H. Franz, and R. Roehlsberger, PETRA III: a low emittance high energy synchrotron radiation source, Acta Crystallogr. Sect. A 61, c137 (2005).

[12] H. Tanaka et al., Stable top-up operation at SPring-8, J. Synchrotron Radiat. 13, 378 (2006).

[13] M. Aiba, P. Beaud, M. Böge, G. Ingold, B. Keil, A. Lüdeke, N. Milas, L. Rivkin, Á. Saá-Hernández, T. Schilcher, V. Schlott, and A. Streun, SLS: Pushing the envelope based on stability, Synchrotron Radiat. News 26, 4 (2013).

[14] A. Lüdeke, Web page of the common operation metrics initiative (2016), https://sites.google.com/site/comi3gls. 